\title{
Carbon Diffusion in Cementite: A Molecular Dynamics Study
}

\author{
E.V. Levchenko ${ }^{a}$, A.V. Evteev ${ }^{b}$, I.V. Belova ${ }^{c}$ and G.E. Murch ${ }^{d}$ \\ Centre for Mass and Thermal Transport in Engineering Materials, \\ Priority Research Centre for Geotechnical and Materials Modelling, \\ School of Engineering, The University of Newcastle, Callaghan, NSW 2308, Australia

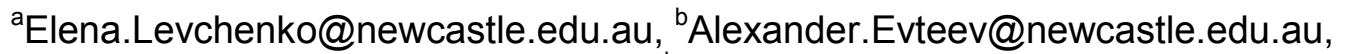 \\ Irina.Belova@newcastle.edu.au, 'Graeme.Murch@newcastle.edu.au
}

Keywords: Carbon diffusion, cementite, atomistic modeling, molecular dynamics.

\begin{abstract}
In this paper, carbon diffusion in cementite is studied by molecular dynamics simulation. An assumption that carbon-carbon interaction occurs only indirectly via neighbouring iron atoms is used. An interstitial mechanism of carbon diffusion in cementite is revealed. The principal tracer diffusion coefficients and activation parameters of carbon diffusion in cementite are calculated for the temperature range 1223-1373 K and compared with the available published experimental data.
\end{abstract}

\section{Introduction}

Cementite has been studied extensively as a component of steel and some cast irons. More recently cementite has attracted a great deal of interest due to its role in carbon nanotube growth [1-3], the metal dusting process [4-8] and in catalytic reactions [9]. However, very little is known, experimentally and theoretically, about the fundamental properties of pure cementite, in particularly about its thermodynamic and diffusion properties. The main reason for this dearth of knowledge comes from the metastability of the compound which causes experimental difficulties e.g. in diffusion studies. For example, conventional radiotracer diffusion experiments in cementite are essentially impossible and chemical diffusion in cementite is problematic in its experimental conditions. Furthermore, it is also difficult to determine the responsible mobile species and atomistic mechanism from the latter experiments. Frequently the properties of cementite have been inferred from measurements on two-phase systems with cementite in an iron matrix [10].

Up to now there are rather few reports about studies on carbon diffusion in cementite. Hillert and Sharp [11] investigated carbon diffusion in cementite at $1138 \mathrm{~K}$ by imposing a fairly low carbon activity of $a_{\mathrm{C}}=1.3$ at the cementite surface. They derived a so-called activity diffusion coefficient of carbon in cementite, which corresponds to a carbon chemical diffusion coefficient in cementite of $\widetilde{D} \approx 1.27 \times 10^{-14} \mathrm{~m}^{2} \mathrm{~s}^{-1}$ [5]. Another group of authors [12-14] utilizing the kinetics of formation of cementite by carburising fine iron particles had estimated the chemical diffusion coefficient of carbon in $\mathrm{Fe}_{3} \mathrm{C}$ as a function of carbon activity (from $a_{\mathrm{C}}=4.3$ up to 20 ) and found it to be in the range of from $10^{-20}$ to $10^{-19} \mathrm{~m}^{2} \mathrm{~s}^{-1}$ at $723 \mathrm{~K}$. Schneider et al. [15] extended the range of carbon activity to 100 and derived the value of the carbon chemical diffusion coefficient in cementite as $\widetilde{D} \approx 5.57 \times 10^{-18} \mathrm{~m}^{2} \mathrm{~s}^{-1}$ at $773 \mathrm{~K}$ using a method similar to [12-14].

The results by Hillert and Sharp [11] were recently reassessed by Hillert et al. [16] using a new method for studying diffusion in interstitial compounds. This method provides very good agreement [16] with the carbon chemical diffusion coefficient in cementite determined by Schneider et al. [15] (the discrepancy is less that $15 \%$ ). Rather than applying a least-squares fit of a straight line to all three values of $\widetilde{D}$ from Hillert and Sharp [11] at $1138 \mathrm{~K}$, Schneider et al. [15] at $773 \mathrm{~K}$ and Ozturk et al. [12] ( $\widetilde{D} \cong$ $2.85 \times 10^{-19} \mathrm{~m}^{2} \mathrm{~s}^{-1}$ ) at $723 \mathrm{~K}$ on the Arrhenius plot, Hillert et al. [16] decided to trust the straight line between two values from experimental information from Schneider et al. [15] at $773 \mathrm{~K}$ and Hillert and Sharp [11] at $1138 \mathrm{~K}$. This gave the result $\widetilde{D}=\widetilde{D}_{0} \exp (-\widetilde{Q} / k T)=1.4 \times 10^{-7} \exp (-1.60 \mathrm{eV} / k T) \mathrm{m}^{2} \mathrm{~s}^{-1}[16]$. An argument for not taking into consideration the data at lower temperature from Ozturk et.al [12] was explained by the fact that compared to other researchers Ozturk and co-workers used iron powder with a less controlled shape and size and so fine that there would be complete carburization within rather short times [16]. 
More recently Schneider and Inden [5] obtained a revised value of the diffusion coefficient as $\widetilde{D} \approx 6.05 \times 10^{-18} \mathrm{~m}^{2} \mathrm{~s}^{-1}$ at $773 \mathrm{~K}$ as well as estimating the activation energy of carbon diffusion in cementite by means of an Arrhenius plot. Using three data points from [5], [11] and [12], the activation energy was found to be $\widetilde{Q} \approx 1.79 \mathrm{eV} /$ atom, which is in good agreement [5] with the previous activation energy estimation by Simkovich et al. [14], where $\widetilde{Q} \approx 1.73 \div 1.92 \mathrm{eV} /$ at. Taking the line as an average through only two points: their result [5] and that of Hillert [11], they obtained a value of the activation energy of carbon diffusion in cementite equal to $\widetilde{Q} \approx 1.59 \mathrm{eV} / \mathrm{at}$. Schneider and Inden [5] supposed that the results by Ozturk et. al [12] ( $\left.\widetilde{D} \cong 2 \times 10^{-19} \mathrm{~m}^{2} \mathrm{~s}^{-1}\right)$ may be taken with some reservation since they did not give any information about the microstructure of the carburized iron powder nor were any measures taken to prevent metal dusting. However, Ozturk et. al [12] applied fairly low C-activities $\left(a_{\mathrm{C}} \leq 20\right)$, which means that the experiments might not be seriously affected by metal dusting [5].

At present, from the experimental data alone, the mechanism of carbon diffusion in cementite remains unknown. Only suggestions have been made about it. For example, in [13] it was tentatively assumed that carbon diffused either by an interstitial or an interstitialcy mechanism because the diffusion coefficient of carbon increased as the activity of carbon was increased.

This paper addresses molecular dynamics (MD) of carbon diffusion in cementite and elucidates the diffusion mechanism of carbon in cementite. Part of the results of this study has been published recently in [17].

\section{The model}

The $\mathrm{Fe}_{3} \mathrm{C}$ structure was simulated as a calculation box with periodic boundary conditions consisting of $10 \times 10 \times 10$ simple orthorhombic unit cells with lattice parameters $a=4.523 \AA, b=$ $5.089 \AA$ and $c=6.743 \AA$ and $4 \mathrm{Fe}$ atoms of type $1,8 \mathrm{Fe}$ atoms of type 2 and $4 \mathrm{C}$ atoms per unit cell [18]. Fe (1) and Fe (2) are two different types of iron sites. This model contains $12000 \mathrm{Fe}$ atoms and $4000 \mathrm{C}$ atoms. The atomic positions are listed in Table 1.

Table 1. The atomic positions in cementite in units of the lattice parameters $a, b$ and $c$, respectively.

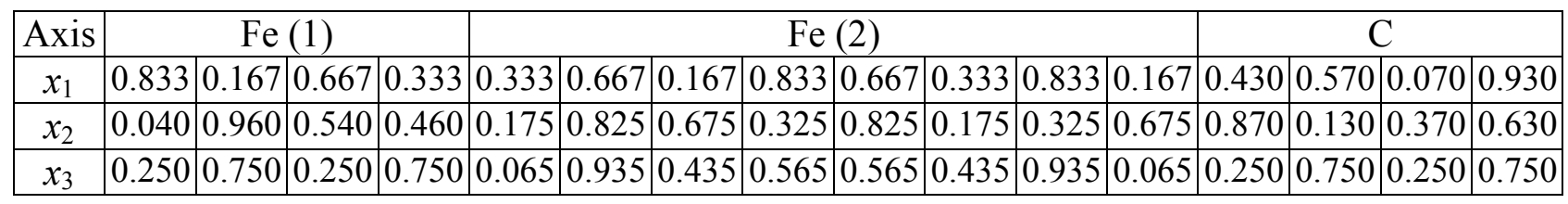

The Fe-Fe interaction was described by the well-known Johnson empirical pair-potential [9]:

$$
\varphi_{\mathrm{Fe}-\mathrm{Fe}}(r)= \begin{cases}-2.195976(r-3.097910)^{3}+2.704060 r-7.436448 \mathrm{eV}, & r<2.40 \AA, \\ -0.639230(r-3.115829)^{3}+0.477871 r-1.581570 \mathrm{eV}, & 2.40 \AA \leq r<3.00 \AA, \\ -1.115035(r-3.066403)^{3}+0.466892 r-1.547967 \mathrm{eV}, & 3.00 \AA \leq r \leq 3.44 \AA .\end{cases}
$$

The Johnson potential and its first derivative smoothly go to zero at a cut-off radius of $3.44 \AA$. The parameters for this potential have been chosen in such a way that the elastic and dynamic properties of $\alpha$-Fe were matched. This potential represents very well the behaviour of Fe interstitials, vacancies and their complexes in $\alpha-\mathrm{Fe}[19,20]$ and $\gamma$-Fe [21].

To describe the Fe-C pair interaction we used the potential proposed by Johnson, Dienes and Damask [22]:

$$
\varphi_{\mathrm{Fe}-\mathrm{C}}(r)=-\varepsilon\left[2\left(\frac{r-r_{\mathrm{c}}}{r_{\mathrm{c}}-r_{0}}\right)^{3}+3\left(\frac{r-r_{\mathrm{c}}}{r_{\mathrm{c}}-r_{0}}\right)^{2}\right] \text {. }
$$

Fitting of the Johnson-Dienes-Damask potential to the experimental data for $\alpha$-Fe (the migration energy of carbon atoms, the activation volume of carbon migration and the vacancy-carbon binding energy) was performed in a set of computer experiments using the Fe-Fe Johnson potential (Eq. 1). Three critical parameters have been changed: the position $r_{0}$, the depth $\varepsilon$ of the potential well and the potential 
cut-off radius $r_{\mathrm{c}}$. The final values for these parameters are: $r_{0}=1.94 \AA, \varepsilon=0.35 \mathrm{eV}, r_{\mathrm{c}}=2.53 \AA$ [22]. The use of the Johnson-Dienes-Damask potential together with the Johnson potential allows for an adequate description of the behaviour of carbon interstitials in $\alpha-\mathrm{Fe}$ [22-24] and in martensite [25]. The structure and relative energy of cementite [12] have also been investigated by means of these potentials. The use of the Johnson-Dienes-Damask potential together with the empirical Pak-Doyama pair potential [26] (this is very similar to the Johnson potential) gives good agreement between the structural characteristics of the MD model of $\mathrm{Fe}-\mathrm{C}$ melts and experimental data [27]. In addition, this combination of potentials provides an explanation of the low glass-forming ability of the metallic alloys of this system compared to metallic Fe-B and Fe-P alloys [27]. More recently, the Johnson-Dienes-Damask potential has been used in a series of studies of carbon nanotube growth on the surface of an iron cluster; see, for example [28].

The situation with the (weak) carbon-carbon potential is more complicated. There is no agreed and detailed data about the preferred type of direct interaction for carbon-carbon pairs in $\mathrm{Fe}-\mathrm{C}$ alloys. That is why as a first approximation we do not consider the direct interaction between carbon-carbon atoms. We do prevent the situation when two carbon atoms occupy the same positions, and, in the present study for describing the C-C interaction, we choose a purely repulsive Born-Mayer potential [29]:

$$
\varphi_{\mathrm{C}-\mathrm{C}}(r)=A \exp \left(-r / r_{\mathrm{s}}\right)
$$

(the energy parameter $A=764.2 \mathrm{eV}$ and the length of screening $r_{\mathrm{s}}=0.219 \AA$ as proposed in [30]) with a cutoff radius of $1.5 \AA$ which is much smaller than the distance between the nearest neighbour carbon atoms.

To initiate the diffusion process, atoms were given initial velocities according to the usual Maxwell distribution at a given temperature, and isothermal annealing procedure was performed in a temperature range of $1223-1373 \mathrm{~K}$ with a step of $50 \mathrm{~K}$. The MD simulations consist of a numerical integration of the equations of atomic motion using a time step $\Delta t=1.5 \times 10^{-15} \mathrm{~s}$ according to the wellknown Verlet algorithm [31]. Periodically, the system was transferred to a state at $T=0 \mathrm{~K}$ where atoms occupied equilibrium positions in a local potential minima. This was done by making use of a static relaxation method. After this, the carbon movements that occurred in the model were analyzed.

\section{Results and discussion}

It was found that carbon diffusion in $\mathrm{Fe}_{3} \mathrm{C}$ is realized by means of interstitial sites, which form a base-centered orthorhombic sublattice in cementite with sublattice parameters $a_{\mathrm{I}}=a, b_{\mathrm{I}}=b$ and $c_{\mathrm{I}}=0.5 c$ and 2 sublattice points $(0.0,0.0,0.0)$ and $(0.5,0.5,0.0)$ in units of $a_{\mathrm{I}}, b_{\mathrm{I}}$ and $c_{\mathrm{I}}$ or, in other words, 4 interstitial positions per $\mathrm{Fe}_{3} \mathrm{C}$ unit cell $(0.0,0.0,0.0),(0.5,0.5,0.0),(0.0,0.0,0.5)$ and $(0.5$, $0.5,0.5$ ) in units of $a, b$ and $c$ (Fig. 1). It should be noted that the distance between the nearest neighbour interstitial sites in [110] and [1 10$]$ directions $(3.404 \AA)$ is very close to the distance between the nearest neighbour interstitial sites in the [001] direction (3.372 $\AA$ ).

The interstitial sites are less energetically favourable for carbon atoms than their original positions. However, with an increase of temperature, because of the increase in entropy, some fraction $\left(C_{\mathrm{I}}\right)$ of the carbon atoms occupies the interstitial sites and, consequently, the same fraction of original carbon positions then becomes vacant. In effect, this is equivalent to the formation of Frenkel defects in ionic compounds especially fluorite related ones. In Fig. 2 we report changes of the fraction of the carbon atoms on original positions during MD isothermal annealing at different temperatures. Analysis of the data in Fig. 2 shows that in the temperature range of $1223-1373 \mathrm{~K}$ these fractions approach 'equilibrium' values after $\sim 13-22$ ns of the annealing.

Using these preliminary results, in order to reveal the most likely movement scenario of carbon atoms in cementite, we calculate first the nearest neighbour distances $(l)$ for all possible carbon atoms jumps in the perfect $\mathrm{Fe}_{3} \mathrm{C}$ structure with the lattice parameters given above. The shortest distances are the distances between the nearest original and interstitial carbon sites with $l^{\prime}=2.547 \AA$ (Fig. 1, dashed lines) and with $l^{\prime \prime}=2.657 \AA$ (Fig. 1, short dashed lines). Every original carbon site has two nearest neighbour interstitial carbon sites of each type. The next two neighbour interstitial carbon sites are located much further; at a distance of $3.151 \AA$. Absolutely the same situation exists for every interstitial carbon site with respect to the original carbon sites (Fig. 1). Other nearest neighbour distances are also considerably longer than $l^{\prime}$ and $l^{\prime \prime}:$ the distance between the nearest neighbour original carbon sites is $3.021 \AA$ and the distance between the 
nearest neighbour interstitial carbon sites is $3.372 \AA$. Therefore, carbon atoms most likely diffuse in cementite by means of a consecutive sequence of jumps: original carbon site $\rightarrow$ interstitial carbon site $\rightarrow$ original carbon site $\rightarrow$... [17].

Next, in the present study three principal tracer diffusion coefficients $D_{1}^{*}, D_{2}^{*}$ and $D_{3}^{*}$ of carbon atoms along three orthogonal crystallographic axes $x_{1}, x_{2}$ and $x_{3}$ at each temperature at 'equilibrium' were calculated using the Einstein equation $2 D_{i}^{*} t=\left\langle\Delta x_{i}^{2}(t)\right\rangle$ using the mean square displacements of the carbon atoms (Fig. 3), $t$ is time and $i=$ $1,2,3$. The determination of principal diffusion coefficients of carbon atoms is very important for such an anisotropic material. To the authors' knowledge this is the first time that the principal diffusion coefficients for carbon diffusion in cementite have been estimated. The tracer diffusion coefficients in different directions are found to be quite different (see Table 2).

In the experiments it is difficult to derive principal diffusion coefficients corresponding to the diffusion along particular crystallographic axis, as well as keep the carbon concentration constant during the time of the experiment. In addition, up to now, there are no experimental data on carbon tracer diffusivities in cementite; however, as was mentioned above, some results for chemical diffusion coefficients of carbon in cementite $\widetilde{D}$ have been obtained experimentally $[5,11-16]$. The results of our recent study of carbon diffusion in cementite have revealed that the thermodynamic factor is quite close to unity (as it will be demonstrated in detail in a separate paper). Thus, if we ignore correlation effects (both tracer and collective) for carbon diffusion in cementite we can consider $\widetilde{D} \sim D^{*}$ and as a result, compare the carbon tracer diffusion coefficients in cementite derived from the present MD study with the experimental carbon chemical diffusion coefficients in cementite.

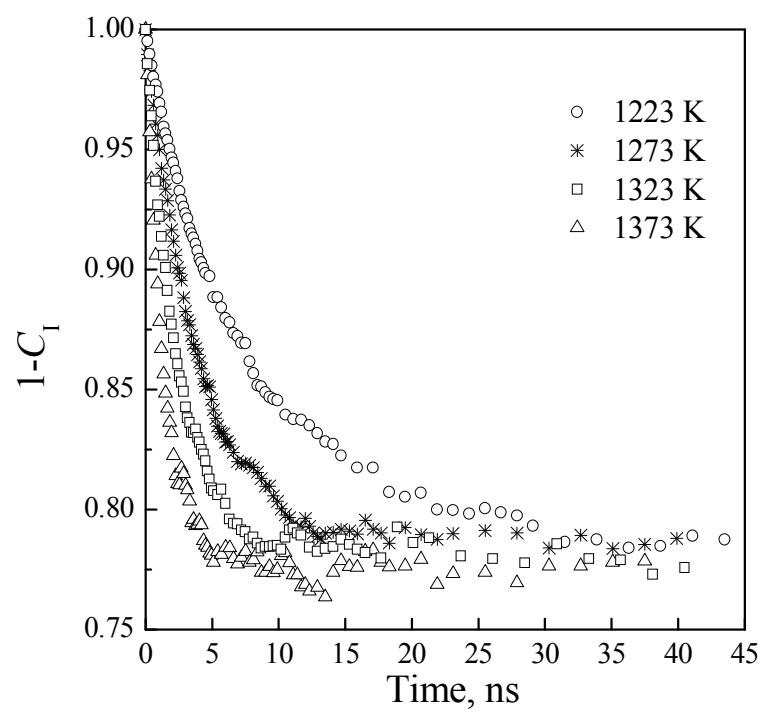

Fig. 2. Changes of the fraction of the carbon atoms on original positions in $\mathrm{Fe}_{3} \mathrm{C}$ during $\mathrm{MD}$ isothermal annealing at different temperatures.

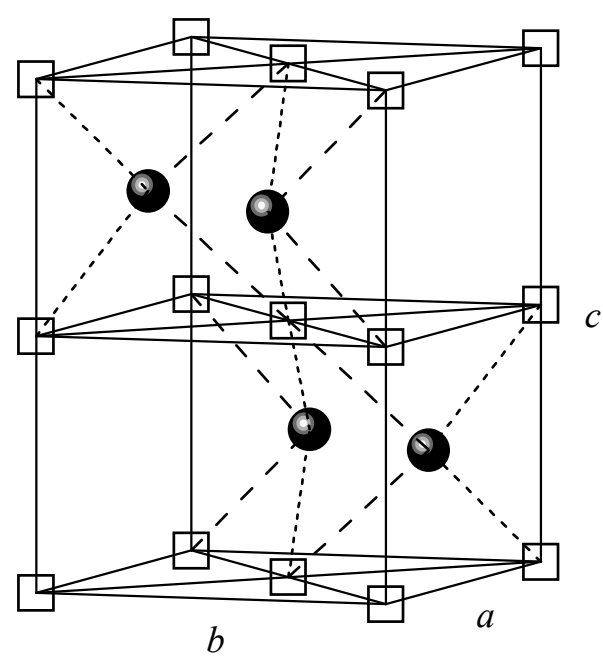

Fig. 1. Arrangement of carbon atoms (spheres) and interstitial sites (squares) in unit cell of $\mathrm{Fe}_{3} \mathrm{C}$ : dashed and short dashed lines show the distances between the first $\left(l^{\prime}=2.547 \AA\right)$ and the second $\left(l^{\prime \prime}=2.657 \AA\right)$ nearest original and interstitial carbon sites, respectively.

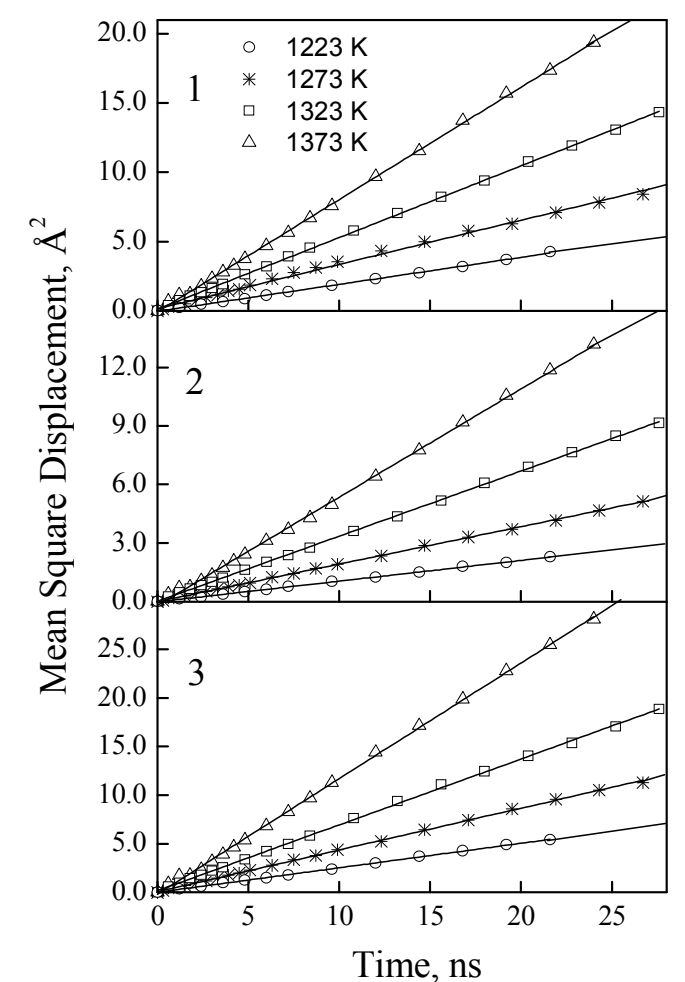

Fig. 3. Mean square displacements of carbon atoms in $\mathrm{Fe}_{3} \mathrm{C}$ along the $x_{1}(1), x_{2}(2)$ and $x_{3}$ (3) principal axes according to the MD simulations. 
Table 2. The principal $D_{i}^{*}$ and average $D^{*}$ tracer diffusion coefficients of carbon atoms in MD model of $\mathrm{Fe}_{3} \mathrm{C}$ and experimental values of chemical diffusion coefficients $\widetilde{D}$ of carbon atoms in $\mathrm{Fe}_{3} \mathrm{C}\left[\right.$ in $\left.10^{-12} \mathrm{~m}^{2} \mathrm{~s}^{-1}\right]$.

\begin{tabular}{|c|l|l|l|l|c|c|}
\hline$T, \mathrm{~K}$ & \multicolumn{1}{|c|}{$D_{1}^{*}$} & \multicolumn{1}{|c|}{$D_{2}^{*}$} & $D_{3}^{*}$ & $D^{*}$ & $\widetilde{D}^{\mathbf{1}}$ & $\widetilde{D}^{\mathbf{2}}$ \\
\hline 1223 & 0.960 & 0.530 & 1.264 & 0.917 & 0.049 & 0.037 \\
\hline 1273 & 1.643 & 0.960 & 2.169 & 1.591 & 0.096 & 0.07 \\
\hline 1323 & 2.620 & 1.672 & 3.436 & 2.576 & 0.178 & 0.114 \\
\hline 1373 & 4.029 & 2.707 & 5.881 & 4.206 & 0.315 & 0.188 \\
\hline
\end{tabular}

${ }^{1}$ Linear extrapolation of approximation via three experimental points [5], [12] and [16]; ${ }^{2}$ - Linear extrapolation of approximation via two experimental points [5] and [16].

If we suppose that the temperature dependence of diffusion coefficient $\widetilde{D}$ is of an Arrhenius type, then extrapolating the experimental data to higher temperatures we get the estimates of the diffusion coefficient $\widetilde{D}$ for the temperatures where our MD calculations were made (Fig. 4). In Table 2 the results of the linear extrapolation of approximation (Fig. 4) via three experimental measurements of $\widetilde{D}$ by Hillert et. al [16] at $1138 \mathrm{~K}$, Schneider and Inden [5] at 773 $\mathrm{K}$, Otzuk et al. [12] at $723 \mathrm{~K}$ as well as through only two high temperature points [5] and [16] are shown. In order to provide the more convenient comparison between the MD and experimental values of the diffusion coefficients the average value of the carbon tracer diffusion coefficient in cementite $D^{*}$ was calculated as the arithmetic mean of the carbon principal tracer diffusion coefficients (Table 2). We can see from the Table 2 and Fig. 4 the tracer diffusion coefficients obtained from MD simulation are one order of magnitude higher than the chemical diffusion coefficients obtained from the experimental data.

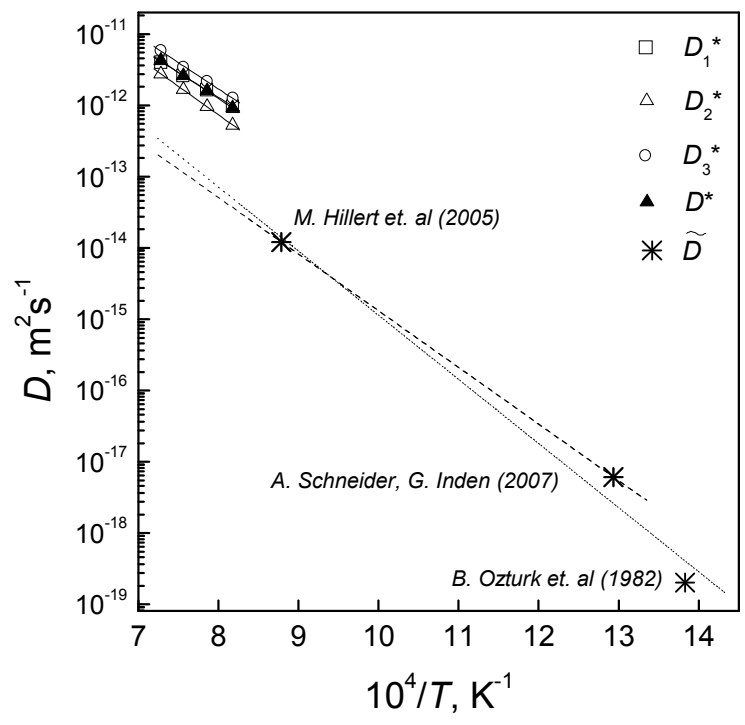

Fig. 4. Arrhenius plot of carbon diffusion coefficients in cementite. Linear approximations via 3 experimental and 2 experimental points as well as via the molecular dynamics points are shown by dotted, dashed and solid lines, respectively.

Assuming an Arrhenius-type dependence of diffusion coefficients of carbon in cementite on temperature (Fig. 4) we can estimate the activation parameters of carbon diffusion in cementite. The activation energies $Q$ and pre-exponential factors $D_{0}$ of carbon tracer diffusion in cementite obtained from the present MD study are shown in Table 3. For comparison the activation energies and pre-exponential factors of carbon chemical diffusion in cementite according to the various experimental works $[5,11,12,14,16]$ are also presented in Table 3.

Table 3. The activation energies $Q$ [in eV/at] and pre-exponential factors $D_{0}$ [in $\mathrm{m}^{2} \mathrm{~s}^{-1}$ ] of carbon diffusion in cementite according to the present MD study and various experimental works.

\begin{tabular}{|c|c|c|c|c|c|c|c|c|c|c|}
\hline Source & $D_{1}^{*}, \mathrm{MD}$ & $D_{2}^{*}, \mathrm{MD}$ & $D_{3}^{*}, \mathrm{MD}$ & $D^{*}, \mathrm{MD}^{2}$ & $\operatorname{Exp} .[5]^{3}$ & Exp.[5] $]^{4}$ & Exp.[14] & Exp. $[16]^{5}$ & Fitt. $1^{6}$ & Fitt. $2^{7}$ \\
\hline$Q$ & & 1.58 & 1.47 & & 1.79 & 1.59 & $173 \div 197$ & 1.60 & 1.79 & 1.57 \\
\hline$D_{0}{ }^{1} \times 10^{7}$ & 4.78 & 17.92 & 13.81 & 9.71 & 13.26 & 1.38 & - & 1.40 & 11.29 & 1.15 \\
\hline
\end{tabular}

${ }^{1}$ All shown pre-exponential factors were calculated in the present work according to the corresponding MD and experimental data, except Exp. [16]. Linear approximations made via: ${ }^{2}-$ the averaged values of $D^{*}$ presented in Table $2 ;{ }^{3}-3$ experimental points [5], [11] and [12]; ${ }^{4}-2$ experimental points [5] and [11]; ${ }^{5}-2$ experimental points [11] and [15]; ${ }^{6}$-Fitting 1: 3 experimental points [5], [12] and [16] (Fig.4 dotted line); ${ }^{7}$ - Fitting 2: 2 experimental points [5] and [16] (Fig.4, dashed line).

In view of the scarcity of the experimental and simulation data it is not possible to determine accurately the activation energy and pre-exponential factor of the carbon diffusion in cementite. Nevertheless, as we can see from Table 3, the agreement between the activation parameters obtained from experimental data and the present MD calculations is quite reasonable. 


\section{Conclusions}

Molecular dynamics simulation has been employed to study carbon diffusion in cementite. The assumption that carbon atoms can interact with each other only indirectly (via neighbouring iron atoms) has been used. The interstitial mechanism of carbon diffusion has been revealed. We have demonstrated that carbon diffusion is realized via interstitial sites, which form four positions per $\mathrm{Fe}_{3} \mathrm{C}$ unit cell $(0.0,0.0$, $0.0),(0.5,0.5,0.0),(0.0,0.0,0.5)$ and $(0.5,0.5,0.5)$ in units of the lattice parameters $a, b$ and $c$. It was argued that carbon diffusion is predominantly a consecutive chain of jumps: original carbon site $\rightarrow$ interstitial carbon site $\rightarrow$ original carbon site $\rightarrow \ldots$

The principal tracer diffusion coefficients and activation parameters of carbon diffusion in cementite have been estimated and compared with the experimental results on carbon chemical diffusion in cementite. The tracer diffusion coefficients from the present molecular dynamics study are one order of magnitude higher than the chemical diffusion coefficients given by the experimental data by reason of the difference in the pre-exponential factors, while the activation parameters obtained from the present molecular dynamics calculations are in good agreement with the experimental data.

\section{Acknowledgements}

This research was supported by the Australian Research Council. One of us (E.V.L.) wishes to thank the University of Newcastle for the award of a Research Fellowship.

\section{References}

[1] A. Maiti, C.J. Brabec, J. Bernholc: Phys. Rev. B Vol. 55 (1997), p. 6097.

[2] A.K. Schaper, H. Hou, A. Greiner, F. Phillipp: J. Catal. Vol. 222 (2004), p. 250.

[3] M. Pérez-Cabero, E. Romeo, C. Royo, A. Monzón et.al: J. Catal. Vol. 224 (2004), p. 197.

[4] D.J. Young: Mat. Sc. Forum Vol. 522-523 (2006), p. 15.

[5] A. Schneider, G. Inden: CALPHAD Vol.31 (2007), p. 141.

[6] E. Pippel, J. Woltersdorf, R. Schneider: Mat. Corr. Vol.49 (1998), p. 309.

[7] P. Szakalos: Mat. Corr. Vol.54 (2003), p. 752.

[8] H.J. Grabke: Mat. Corr. Vol. 54 (2003), p. 736.

[9] W. Arabczyk, W. Konicki, U. Narkiewicz, I. Jasinska et.al: Appl. Catal. A Vol. 266 (2004), p. 135.

[10] J.Hägland and G.Grimvall: Phys.Rev. B Vol. 44/7 (1991), p. 2914.

[11] M. Hillert, R.D. Sharp: Jernkont. Ann. Vol. 137 (1953), p. 785.

[12] B. Ozturk, V.L.Fearing, J.A. Ruth, G.Simkovich: Metall. Trans. A Vol.13 (1982), p. 1871.

[13] B. Ozturk, V.L.Fearing, J.A. Ruth, G.Simkovich: Solid State Ion. Vol.12 (1984), p. 145.

[14] G.Simkovich, in: Selected topics in High Temperature Chemistry, edited by O. Johannson, Elsevier Science Publishers, Amsterdfam (1989).

[15] A. Schneider, G. Inden, H.J. Grabke, in: Interface Controlled Materials (EUROMAT 99), edited by M. Rühle, H. Gleiter, Wiley-VCH, Weinheim (2000).

[16] M. Hillert, L.Hägland, J. Ågren: J. Appl. Phys. Vol. 98 (2005), p. 053511.

[17] A.V. Evteev, E.V. Levchenko, I.V. Belova, G.E. Murch: Defect and Diffus. Forum Vol. 277 (2008), p. 97.

[18] R.Wykoff: Crystal Structures (Interscience, New York 1964).

[19] R.A. Johnson: Phys. Rev. Vol. 134 (1964), p. A1329.

[20] J.R. Beeler, Jr. and R.A. Johnson: Phys. Rev. Vol. 156 (1967), p. 677.

[21] R.A. Johnson: Phys. Rev. Vol. 145 (1966), p. 423.

[22] R.A. Johnson, G.J. Dienes and A.C. Damask: Acta Metall. Vol.12 (1964), p. 1215.

[23] R.A. Johnson, A.C. Damask: Acta Metall. Vol. 12 (1964), p. 443.

[24] R.A. Johnson: Acta Metall. Vol. 15 (1967), p. 513.

[25] R.A. Johnson: Acta Metall. Vol. 13 (1965), p. 1259.

[26] H.M. Pak, M. Doyama: J. Fac.Eng. Univ. Tokyo, Vol. B 30 (1969), p. 111.

[27] A.V. Evteev, A.T. Kosilov, and E.V. Levtchenko: Acta Mater. Vol.51 (2003), p. 2665.

[28] F. Ding, A. Rosen and K. Bolton: J. Chem. Phys. Vol. 121 (2004), p. 2775.

[29] W. Eckstein: Computer Simulation of Ion-Solid Interactions (Springer-Verlag, Berlin 1991).

[30] H.H. Andersen and P. Sigmund: J. Nucl. Instr. Meth. Vol. 38 (1965), p. 238.

[31] L. Verlet: Phys. Rev. Vol.159 (1967), p. 98. 
Diffusion in Solids and Liquids IV

doi:10.4028/www.scientific.net/DDF.283-286

Carbon Diffusion in Cementite: A Molecular Dynamics Study doi:10.4028/www.scientific.net/DDF.283-286.24 